\title{
ON THE EXTENSION OF OPERATORS WITH RANGE IN A $C(K)$ SPACE
}

\author{
JORAM LINDENSTRAUSS ${ }^{1}$
}

1. Introduction. Let $K$ be a compact Hausdorff space and let $C(K)$ be the space of all the continuous real-valued functions on $K$ with the supremum norm. The purpose of this note is to prove the following

THEOREM. For $X=C(K)$ the following four statements are equivalent.

(i) For every two Banach spaces $Z \supset Y$ with $\operatorname{dim}(Z / Y)=1$ and every operator $T$ from $Y$ into $X$ with a separable range there is an extension $\widetilde{T}$ of $T$ from $Z$ into $X$ with $\|\tilde{T}\|=\|T\|$.

(ii) For every two Banach spaces $Z \supset Y$ with $\operatorname{dim} Y=2, \operatorname{dim} Z=3$ and every operator $T$ from $Y$ into $X$ there is an extension $\tilde{T}$ of $T$ from $Z$ into $X$ with $\|\tilde{T}\|=\|T\|$.

(iii) There is a $\lambda<2$ such that for every two Banach spaces $Z \supset Y$ with $\operatorname{dim}(Z / Y)=1$ and every operator $T$ from $Y$ into $X$ with a separable range there is an extension $\tilde{T}$ of $T$ from $Z$ into $X$ with $\|\tilde{T}\| \leqq \lambda\|T\|$.

(iv) $K$ is an F-space in the terminology of Gillman and Jerison [4, p. 208]. That is, for every $f \in C(K)$ there is a $g \in C(K)$ such that $f(k)>0$ implies $g(k) \geqq 1$ and $f(k)<0$ implies $g(k) \leqq-1$.

Assuming the continuum hypothesis the following statement is also equivalent to the preceding ones.

(v) For every two Banach spaces $Z \supset Y$ and every operator $T$ from $Y$ into $X$ with a separable range there is an extension $\tilde{T}$ of $T$ from $Z$ into $X$ with $\|\tilde{T}\|=\|T\|$.

The equivalence of (i) and (iv) is essentially due to Aronszajn and Panitchpakdi [2].

Our main tool in the proof of the theorem will be the equivalence of each of (i), (ii) and (iii) with intersection properties of the cells in $X$. The connection between extension and intersection properties was first observed by Nachbin in [13] and then used in more general situations in [2], [7] and [11].

We consider only Banach spaces over the reals. By "operator" we always mean "bounded linear operator." The cell $\left\{x ;\left\|x-x_{0}\right\| \leqq r\right\}$ is denoted by $S\left(x_{0}, r\right)$.

Presented to the Society November 9, 1962; received by the editors December 14, 1962.

${ }^{1}$ Research supported by NSF Grant 25222. 
2. Proof of the theorem. From the proof of Theorem 1.8 in [11] it follows that a Banach space $X$ satisfies (i) if and only if for every collection of mutually intersecting cells in $X$, whose centers generate a separable subspace, there is a point in $X$ common to all the cells in the collection. Aronszajn and Panitchpakdi [2, §5, Theorem 2] showed that a $C(K)$ space has this intersection property if and only if $K$ satisfies a certain topological condition which, as remarked by Henriksen [8], is equivalent to (iv). Hence (i) $\Leftrightarrow$ (iv).

From the proof of Theorem 1.8 in [11] it follows also that a Banach space $X$ satisfies (ii) if and only if for every collection of mutually intersecting cells in $X$, whose centers generate a 2-dimensional subspace, there is a point in $X$ common to all the cells in the collection. This fact is used in the following lemma, which is the essential step in the proof that (ii) implies (iv).

Lemma. Suppose $X=C(K)$ satisfies (ii) and let $f \in C(K)$. Let $\alpha_{n}, \beta_{n}$, $\gamma_{n}, \delta_{n}$ be sequences of real numbers tending to 0 and satisfying

$$
\alpha_{n+1}<\beta_{n}<\alpha_{n}, \quad \gamma_{n}<\delta_{n}<\gamma_{n+1}, \quad n=1,2, \cdots .
$$

Then there is a $g \in C(K)$ such that for every $n$

$$
\begin{aligned}
& \beta_{n} \leqq f(k) \leqq \alpha_{n} \Rightarrow g(k) \geqq 1, \\
& \gamma_{n} \leqq f(k) \leqq \delta_{n} \Rightarrow g(k) \leqq-1 .
\end{aligned}
$$

Proof. Let $\tau_{a, b, c, d}(t)$, where $a<b<c<d$, denote the trapezoidal function equal to 0 for $t \leqq a$ and $t \geqq d$, equal to 1 for $t \in[b, c]$ and linear in $[a, b]$ and $[c, d]$. Put

$$
\tau_{2 n-1}(t)=\tau_{\alpha_{n+1}, \beta_{n}, \alpha_{n}, \beta_{n-1}}(t), \quad \tau_{2 n}(t)=\tau_{\delta_{n-1}, \gamma_{n}, \delta_{n}, \gamma_{n+1}}(t),
$$

for $n=1,2, \cdots$ (where $\beta_{0}$ is any number $>\alpha_{1}$ and $\delta_{0}<\gamma_{1}$ ). Clearly

$$
\sum_{n=1}^{\infty} \tau_{n}(t)=1, \quad t \in\left[\gamma_{1}, \alpha_{1}\right], t \neq 0
$$

Hence if $\lambda_{n} \rightarrow \lambda$ the function equal to $\sum \lambda_{n} \tau_{n}(t)$ for $t \neq 0$ and equal to $\lambda$ if $t=0$ is continuous. In particular

$$
\begin{array}{ll}
u(t)=\sum_{n=1}^{\infty} \sin n^{-1} \cdot \tau_{n}(t), & t \neq 0, u(0)=0 \\
v(t)=\sum_{n=1}^{\infty} \cos n^{-1} \cdot \tau_{n}(t), & t \neq 0, v(0)=1
\end{array}
$$

are continuous. Let $m$ be any integer and let $\lambda_{m}$ be defined by 


$$
\begin{aligned}
\lambda_{m}^{-1} & =\left(1-\max _{p \neq m} \cos \left(p^{-1}-m^{-1}\right)\right) / 2 \\
& =\left(1-\cos \left(m^{-1}(m+1)^{-1}\right)\right) / 2 .
\end{aligned}
$$

Consider the function

$$
\phi_{m}(t)=(-1)^{m+1} \lambda_{m}\left(\sin m^{-1} \cdot u(t)+\cos m^{-1} \cdot v(t)\right) .
$$

For $m=2 n-1$ we have

(3) $\quad \phi_{2 n-1}(t)=\max _{-\infty \leq s \leq \infty}\left|\phi_{2 n-1}(s)\right|=\lambda_{2 n-1} \quad$ for $\beta_{n} \leqq t \leqq \alpha_{n}$, and also, by (1) and (2),

$$
\phi_{2 n-1}(t) \leqq \lambda_{2 n-1}-2 \quad \text { for } t \leqq 0 .
$$

Similarly for $m=2 n$

$$
\phi_{2 n}(t)=-\max _{-\infty \leq 8 \leq \infty}\left|\phi_{2 n}(s)\right|=-\lambda_{2 n} \quad \text { for } \gamma_{n} \leqq t \leqq \delta_{n}
$$

(6) $\phi_{2 n}(t) \geqq-\left(\lambda_{2 n}-2\right)$ for $t \geqq 0$.

By (3), (4), (5) and (6)

(7) $\left|\phi_{m}(t)-\phi_{p}(t)\right| \leqq \lambda_{m}+\lambda_{p}-2 \quad$ for every $m, p$ and $t$.

Put $\psi_{m}(k)=\phi_{m}(f(k)), m=1,2, \ldots$ Each $\psi_{m}$ belongs to the 2-dimensional subspace of $C(K)$ spanned by $u(f(k))$ and $v(f(k))$. By $(7)$ the cells $S\left(\psi_{m}, \lambda_{m}-1\right)$ are mutually intersecting and hence by our assumption on $X$ there is a $g \in X$ with $\left\|g-\psi_{m}\right\| \leqq \lambda_{m}-1, m=1,2, \cdots$. By (3) and (5)

$$
\begin{aligned}
& \beta_{n} \leqq f(k) \leqq \alpha_{n} \Rightarrow \psi_{2 n-1}(k)=\lambda_{2 n-1} \Rightarrow g(k) \geqq 1, \\
& \gamma_{n} \leqq f(k) \leqq \delta_{n} \Rightarrow \psi_{2 n}(k)=-\lambda_{2 n} \Rightarrow g(k) \leqq-1,
\end{aligned}
$$

and this concludes the proof of the lemma.

We show now that (ii) $\Rightarrow$ (iv). Let $C(K)$ satisfy (ii) and let $f \in C(K)$ with $\|f\|=1$. By the lemma there are $g^{\prime}, g^{\prime \prime} \in C(K)$ such that for $n=1,2, \cdots$

$$
\begin{aligned}
-1 /(2 n-1) & \leqq f(k) \leqq-1 / 2 n \Rightarrow g^{\prime}(k) \leqq-1, \quad g^{\prime \prime}(k) \leqq-1, \\
1 /(2 n+1) & \leqq f(k) \leqq 1 / 2 n \Rightarrow g^{\prime}(k) \geqq 1, \\
1 / 2 n & \leqq f(k) \leqq 1 /(2 n-1) \Rightarrow g^{\prime \prime}(k) \geqq 1 .
\end{aligned}
$$

Hence $g_{1}=\max \left(g^{\prime}, g^{\prime \prime}\right)$ satisfies

$f(k)>0 \Rightarrow g_{1}(k) \geqq 1, \quad-1 /(2 n-1) \leqq f(k) \leqq-1 / 2 n \Rightarrow g_{1}(k) \leqq-1$,

$$
n=1,2, \cdots \text {. }
$$


In a similar manner it follows that there is a $g_{2} \in C(K)$ satisfying

$$
\begin{array}{r}
f(k)>0 \Rightarrow g_{2}(k) \geqq 1, \quad-1 / 2 n \leqq f(k) \leqq-1 /(2 n+1) \Rightarrow g_{2}(k) \leqq-1, \\
n=1,2, \cdots .
\end{array}
$$

The function $g=\min \left(g_{1}, g_{2}\right)$ has the property required in (iv). Hence (ii) $\Rightarrow$ (iv).

A Banach space $X$ has property (iii) with a certain $\lambda$ if and only if for every collection of mutually intersecting cells $\left\{S\left(x_{\alpha}, r_{\alpha}\right)\right\}_{\alpha \in A}$ in $X$ such that the set $\left\{x_{\alpha}\right\}_{\alpha \in A}$ generates a separable subspace, there is an $x \in X$ satisfying $\left\|x-x_{\alpha}\right\| \leqq \lambda r_{\alpha}$ for every $\alpha \in A$ (cf. the proofs of [7, Theorem $1 ; 11$, Theorem 1.8]). Let $C(K)$ satisfy (iii) and let $f \in C(K)$. Further let $\tau_{n}(t)$ be equal to 1 for $t \geqq 1 / n$, equal to -1 for $t \leqq-1 / n$ and linear in $[-1 / n, 1 / n]$. The cells $\left\{S\left(\tau_{n}(f), 1 / 2\right)\right\}_{n=1}^{\infty}$ are mutually intersecting. Let $g \in \bigcap_{n} S\left(\tau_{n}(f), \lambda / 2\right)$. Since $\lambda<2$ it follows that

$$
f(k)>0 \Rightarrow g(k) \geqq 1-\lambda / 2, \quad f(k)<0 \Rightarrow g(k) \leqq-1+\lambda / 2 .
$$

Hence $K$ satisfies (iv).

Since the implications (i) $\Rightarrow$ (ii) and (i) $\Rightarrow$ (iii) are trivial we have already shown that the statements (i)-(iv) are equivalent.

To conclude the proof of the theorem we show that, assuming the continuum hypothesis, $(i) \Rightarrow(v)$. This part of the proof is valid for general Banach spaces $X$. Let $X$ satisfy (i), let $Z \supset Y$ and let $T$ be an operator from $Y$ into $X$ with a separable range. The closed subspace $Y_{0}$ of $X$ generated by $T Y$ is separable. Hence $Y_{0}$ is isometric to a subspace of $m$. Since the operator $T$ from $Y$ into $Y_{0}$ has a norm preserving extension from $Z$ into $m[3$, p. 94], we have only to show that the identity map from $Y_{0}$ into $X$ has a norm preserving extension from $m$ into $X$. Hence we may assume that $Y$ is separable and $Z=m$. By the continuum hypothesis the cardinality of $m$ is $\aleph_{1}$. Let $\left\{z_{\alpha}\right\}_{\alpha \in \Omega}$ be a well ordering of the points of $m$, where $\Omega$ is the set of all ordinals smaller than the first uncountable one. For every $\alpha$ let $Z_{\alpha}$ be the closed subspace of $m$ spanned by $Y$ and $\left\{z_{\beta}\right\}_{\beta<\alpha} . Z_{\alpha}$ is separable for every $\alpha$. We now construct inductively for every $\alpha \in \Omega$ an operator $T_{\alpha}$ from $Z_{\alpha}$ into $X$ such that $T_{1}=T,\left\|T_{\alpha}\right\|=\|T\|$, and the restriction of $T_{\alpha}$ to $Z_{\beta}(\beta<\alpha)$ is equal to $T_{\beta}$. For $\alpha=\alpha^{\prime}+1$ let $T_{\alpha}$ be any norm preserving extension of $T_{\alpha^{\prime}}$ to $Z_{\alpha}$. Such an extension exists by (i) $\left(\operatorname{dim}\left(Z_{\alpha} / Z_{\alpha^{\prime}}\right)\right.$ is either 0 or 1$)$. If $\alpha$ is a limiting ordinal we define $T_{\alpha}$ first on $\bigcup_{\beta<\alpha} Z_{\beta}$ by $T_{\alpha} z=T_{\beta} z$ if $z \in Z_{\beta}, \beta<\alpha$ (the definition is easily seen to be independent of the choice of $\beta$ ), and extend it by continuity to $Z_{\alpha}$, which is the closure of $\bigcup_{\beta<\alpha} Z_{\beta}$. Having constructed the $T_{\alpha}$, $\alpha \in \Omega$, we define $\tilde{T}$ on $m\left(=\bigcup_{\alpha \in \Omega} Z_{\alpha}\right)$ by $\tilde{T} z=T_{\alpha} z$ if $z \in Z_{\alpha} . \tilde{T}$ is clearly 
a norm preserving extension of $T$ from $m$ into $X$. This concludes the proof of the theorem.

3. Remarks. 1. If we do not restrict ourselves to $C(K)$ spaces $X$, (i), (ii) and (iii) are no longer equivalent. $c_{0}$, for example, satisfies (ii) $[12$, Chapter 2, §6] but not (iii) (cf. Sobczyk [14]). Every finitedimensional space satisfies (iii) $[7$, p. 198] but in general it does not satisfy (ii).

2. The fact that (i) and (iii) are equivalent for $C(K)$ spaces is similar to a result, due to Amir [1] and Isbell and Semadeni [9], that if a $C(K)$ space has a projection constant $\lambda<2$ then it has already the projection constant 1 (i.e., $K$ is extremally disconnected).

3. The requirement that the norm of the extension $\tilde{T}$ is exactly equal to that of $T$ is essential in (ii). Grothendieck [6] proved that every $C(K)$ space $X$ has the following extension property: For every two Banach spaces $Z \supset Y$ and every compact operator $T$ from $Y$ into $X$ there is, for every $\epsilon>0$, a compact extension $\widetilde{T}$ of $T$ from $Z$ into $X$ with $\|\widetilde{T}\| \leqq(1+\epsilon)\|T\|$. (Cf. also [10], [12] for characterizations of the spaces $X$ having this extension property.)

4. In property (iii) we cannot take $\lambda=2$. Indeed, for every two Banach spaces $Z \supset Y$ with $\operatorname{dim} Z / Y=1$ and every operator $T$ from $Y$ into a $C(K)$ space $X$ there is an extension $\widetilde{T}$ of $T$ from $Z$ into $X$ with $\|\tilde{T}\| \leqq 2\|T\|$. As shown by Grünbaum [7] this assertion is equivalent to the following: For every collection of mutually intersecting cells $\left\{S\left(f_{\alpha}, r_{\alpha}\right)\right\}_{\alpha \in A}$ in a $C(K)$ space $\bigcap_{\alpha \in A} S\left(f_{\alpha}, 2 r_{\alpha}\right) \neq \varnothing$. That a $C(K)$ space has this intersection property follows immediately from the following two statements. (a) Let $X$ be a Banach space and let $\left\{S\left(x_{\alpha}, r_{\alpha}\right)\right\}_{\alpha \in A}$ be a collection of mutually intersecting cells in $X$. Then for every $\epsilon>0, \bigcap_{\alpha \in A} S\left(x_{\alpha}, 2 r_{\alpha}+\epsilon\right) \neq \varnothing$. (b) Let $X$ be a $C(K)$ space and let $\left\{S\left(f_{\alpha}, r_{\alpha}\right)\right\}_{\alpha \in A}$ be a collection of cells in $X$. If for every $\epsilon>0, \bigcap_{\alpha \in A} S\left(f_{\alpha}, r_{\alpha}+\epsilon\right) \neq \varnothing$, then also $\bigcap_{\alpha \in A} S\left(f_{\alpha}, r_{\alpha}\right) \neq \varnothing$. Statement (a) was proved by Grünbaum [7, p. 198]. Actually he only asserts that (with the same notation as in (a)) $\bigcap_{\alpha \in A} S\left(x_{\alpha},(2+\epsilon) r_{\alpha}\right) \neq \varnothing$, but his proof shows that also (a) holds. Statement (b) follows from the following observation (contained implicitly in the proof of Theorem 2 in $\$ 5$ of [2]): $\bigcap_{\alpha \in A} S\left(f_{\alpha}, r_{\alpha}\right) \neq \varnothing$ if and only if for every $k_{0} \in K$

$$
\left.\lim _{\boldsymbol{k} \rightarrow \boldsymbol{k}_{0}} \sup _{\boldsymbol{\alpha}}\left(f_{\alpha}(k)-\boldsymbol{r}_{\alpha}\right) \leqq \lim _{\boldsymbol{k} \rightarrow \boldsymbol{k}_{0}} \inf _{\boldsymbol{\alpha}} \inf _{\boldsymbol{\alpha}}(k)+\boldsymbol{r}_{\alpha}\right),
$$

where $\lim \sup _{k \rightarrow k_{0}}$ denotes the inf, over the neighborhoods $G$ of $k_{0}$, of $\sup _{k \in G} . \lim \inf _{k \rightarrow k_{0}}$ is defined analogously.

In the terminology of Grünbaum [7] this remark (together with a result of Amir [1]) means that the expansion constant of a $C(K)$ 
space is exact, being 1 for extremally disconnected $K$ and 2 in all other cases.

5. The range of the extension $\tilde{T}$ in property (v) will not in general be separable. For example, if $T$ is the identity map from a separable nonreflexive subspace $Y$ of $X$, into $X$, and if $Z=m(\supset Y)$, then every extension $\widetilde{T}$ of $T$ from $Z$ into $X$ has a nonseparable range. This follows from a theorem of Grothendieck [5] which asserts that every bounded operator from $m$ into a separable space is weakly compact.

6. Let $\mathfrak{m}$ be an infinite cardinal and let (i-m), (iii- $\mathfrak{m})$ and $(v-\mathfrak{m})$ be the properties obtained from (i), (iii) and (v) by replacing the requirement that $T$ has a separable range by the requirement that the range of $T$ has a dense set of cardinality at most $m$. As in the proof of the theorem it can be shown that for $C(K)$ spaces (i-m) is equivalent to (iii-m) and that if we assume the generalized continuum hypothesis, (i-m) and $(v-\mathfrak{m})$ are equivalent for general Banach spaces $X$.

4. Appendix. We shall prove now that the Banach spaces $X=C(K)$ with $K$ an $F$ space (we assume always that $K$ is compact Hausdorff) have also the following extension property

(vi) For every two Banach spaces $Z$ and $Y$, with $Z \supset X$ and $Y$ separable, and every operator $T$ from $X$ into $Y$ there is a norm preserving extension of $T$ from $Z$ into $Y$.

This is the "from" extension property corresponding to the "into" property (v). We assume the continuum hypothesis. The assertion stated above is an immediate consequence of Proposition 2 below.

Proposition 1. Property (vi) is equivalent to each of the following two properties.

(vii) $X^{*}$ is an $L_{1}$ space, and for every two Banach spaces $Z$ and $Y$, with $Z \supset X$ and $Y$ separable, and every operator $T$ from $X$ into $Y$ there is an extension of $T$ from $Z$ into $Y$.

(viii) $X^{*}$ is an $L_{1}$ space, and every operator from $X$ into a separable Banach space is weakly compact.

Proof. (vi) $\Rightarrow$ (vii) follows from $(8) \Rightarrow(2)$ in Theorem 1 of $[10]$ (the assumption in (8) there that $X$ has the metric approximation property can be discarded. This was shown in our paper "On the extension of operators with a finite-dimensional range," submitted to the Illinois J. Math.). (vii) $\Rightarrow$ (viii) follows from the fact that, for every set $I$, every operator from $m(I)$ (=the space of all bounded real-valued functions on $I$ ) to a separable space is weakly compact (Grothendieck 
[5]). We apply (vii) in the case $Z$ is an $m(I)$ space containing $X$ (clearly such a $Z$ always exists). (viii) $\Rightarrow$ (vi) follows from $(2) \Rightarrow(9)$ in Theorem 1 of [10].

Proposition 2. Property (v) implies (vi).

Proof. Let $X$ satisfy (v). That $X^{*}$ is an $L_{1}$ space was proved by Grothendieck [6] (cf. (3) $\Rightarrow(2)$ in Theorem 1 of [10]). Let $Y$ be a separable Banach space and let $T$ be an operator from $X$ into $Y$. We show that $T$ is weakly compact. It is sufficient to show that the restriction of $T$ to every separable subspace of $X$ is weakly compact. Let $X_{0}$ be a separable subspace of $X$. By (v) there is an operator $T_{0}$ from $m\left(\supset X_{0}\right)$ into $X$ whose restriction to $X_{0}$ is the identity. By the result of Grothendieck [5], cited in the proof of Proposition 1, $T T_{0}$ is weakly compact. It follows that the restriction of $T T_{0}$, and hence of $T$, to $X_{0}$ is also weakly compact.

We have shown, in particular, that every $C(K)$ space with $K$ an $F$ space has property (viii). This generalizes a result of Grothendieck in [5], where this assertion was proved for extremally disconnected $K$. Semadeni (in an unpublished note ${ }^{2}$ ) remarked that the proof of Grothendieck actually holds for every basically disconnected $K$ (cf. [4, p. 22] for the definition of this notion). There exist connected $F$ spaces [4, p. 211] and thus our result solves problem 9 of [9].

Concluding, we remark that there are $C(K)$ spaces satisfying (vi) without $K$ being an $F$ space. This follows from examples given in [1] and [9] and the fact that if $C\left(K_{1}\right)$ is isomorphic to $C\left(K_{2}\right)$ (as a Banach space) and if $C\left(K_{1}\right)$ satisfies (vii) then also $C\left(K_{2}\right)$ satisfies (vii) and hence (vi). It would be interesting to have a characterization of all spaces $K$ for which $C(K)$ satisfies (vi).

\section{REFERENCES}

1. D. Amir, Continuous function spaces with the bounded extension property, Bull. Res. Council Israel Sect. F 10 (1962), 133-138.

2. N. Aronszajn and P. Panitchpakdi, Extensions of uniformly continuous transformations and hyperconvex metric spaces, Pacific J. Math. 6 (1956), 405-441.

3. M. M. Day, Normed linear spaces, Springer, Berlin, 1958.

4. L. Gillman and M. Jerison, Rings of continuous functions, Van Nostrand, New York, 1960.

5. A. Grothendieck, Sur les applications linéaires faiblement compactes d'espaces $d u$ type $C(K)$, Canad. J. Math. 5 (1953), 129-173.

6. - Résumé de la théorie métrique des produits tensoriels topologiques, Bol. Soc. Mat. São Paulo 8 (1956), 1-79.

7. B. Grünbaum, Some applications of expansion constants, Pacific J. Math. 10 (1960), 193-201.

${ }^{2}$ Cf. also [9, Proposition 8]. 
8. M. Henriksen, Some remarks on a paper of Aronszajn and Panitchpakdi, Pacific J. Math. 7 (1957), 1619-1621.

9. J. R. Isbell and Z. Semadeni, Projection constants and spaces of continuous functions, Trans. Amer. Math. Soc. 107 (1963), 38-48.

10. J. Lindenstrauss, On the extension property for compact operators, Bull. Amer. Math. Soc. 68 (1962), 484-487.

11. - Extension of compact operators. II, Technical note no. 31, Jerusalem, June 1962; Trans. Amer. Math. Soc. (to appear).

12. - Extension of compact operators. III, Technical note no. 32, Jerusalem, July 1962; Trans. Amer. Math. Soc. (to appear).

13. L. Nachbin, A theorem of the Hahn-Banach type for linear transformations, Trans. Amer. Math. Soc. 68 (1950), 28-46.

14. A. Sobczyk, Projections of the space $m$ on its subspace $c_{0}$, Bull. Amer. Math. Soc. 47 (1941), 938-947.

YALE UNIVERSITY

\section{ON THE STRUCTURE OF THE GREEN'S OPERATOR}

\section{ROBERT CARROLL ${ }^{1}$}

1. Introduction. In the study of Cauchy problems of the form

$$
d u / d t+A u=f ; \quad u(\tau)=T
$$

(where for example: $t \rightarrow u(t) \in \mathcal{E}^{1}(H)$ on $(\tau, b] ; t \rightarrow u(t) \in \mathcal{E}^{0}(D(A))$ on $[\tau, b] ; H$ is a Hilbert space; $-A$ is a closed (unbounded) operator, infinitesimal generator of a strongly continuous semi-group; $\mathcal{E}^{k}(H)$ is the space of $k$-times continuously differentiable functions of $t$ with values in $H$; the domain of $A, D(A)$, has the graph topology; and $f, T$ are suitable), the solution takes the appearance

$$
u(t)=G(t, \tau) u(\tau)+\int_{\tau}^{t} G(t, \xi) f(\xi) d \xi .
$$

Formally the Green's operator $G(t, \xi)$ may be written $G(t, \xi)$ $=\exp [-A(t-\xi)]$ (for general results in this direction see for example $[1 ; 2 ; 3])$. In this article we propose to study representations related to (1.2) for solutions of general operational differential equations $S u=f$ (the operators need not be differential operators of course but therein lies the motivation, see $[4 ; 5]$; cf. also the papers $[3 ; 6 ; 7 ; 8$; 9; 10]).

2. Basic framework. Let $H$ be a Hilbert space and $\left(S_{0}, S_{0}^{\prime}\right)$ a formally adjoint pair of closed densely defined operators in the sense

Presented to the Society, April 19, 1963; received by the editors February 7, 1963.

1 Research supported in part by the Rutgers Research Council. 